GLOBAL CHALLENGES

\section{One gene, lots of rice}

Understanding the genetic basis of crop yield has been thought to require the identification of many genes of small effect. New research has uncovered an important exception to this rule - a rice locus that has a major effect on several aspects of yield.

Many QTLs have already been identified for aspects of rice yield, such as the number of grains per plant, plant height and flowering time. Zhang and colleagues focused on one region of chromosome 7 that has been implicated in these studies and which simultaneously affects all three of these traits. In a series of crosses and backcrosses they found no recombination between the three traits, implying that they are controlled by a single locus.

Using molecular markers, they narrowed the chromosomal region down to one that contains 450 genes. When they sequenced a part of this region from their two parental strains, they found a deletion in one parent that spanned a region containing a gene with a domain that is also found in genes that affect flowering time in Arabidopsis thaliana. Expressing this gene - which they called Gdh7 (grain number, plant height and heading date 7) - in plants with low yield, short height and early flowering was sufficient to transform all three phenotypes.
A survey of natural populations showed that the allele that increases yield, height and time to flowering is found in tropical and subtropical populations with long growing seasons, whereas various alleles that reduce the time to flowering predominate in regions with shorter seasons. This highlights how manipulating this major-effect locus could be used to optimize rice cultivars for the regions that they are grown in and for changing climate conditions.

Patrick Goymer

ORIGINAL RESEARCH PAPER Xue, W. $\varepsilon$

Xing, Y. et al. Natural variation in Gdh7 is an important regulator of heading time and yield potential in rice. Nature Genet. 4 May 2008 (doi:10.1038/ng.143)

FURTHER READING Jung, K.-H., An, G. \& Ronald, P. C. Towards a better bowl of rice: assigning function to tens of thousands of rice genes. Nature Rev. Genet. 9, 91-101 (2008)| Takeda, S. \& Matsuoka, M. Genetic approaches to crop improvement: responding to environmental and population changes. Nature Rev. Genet. 9, 444-457 (2008)

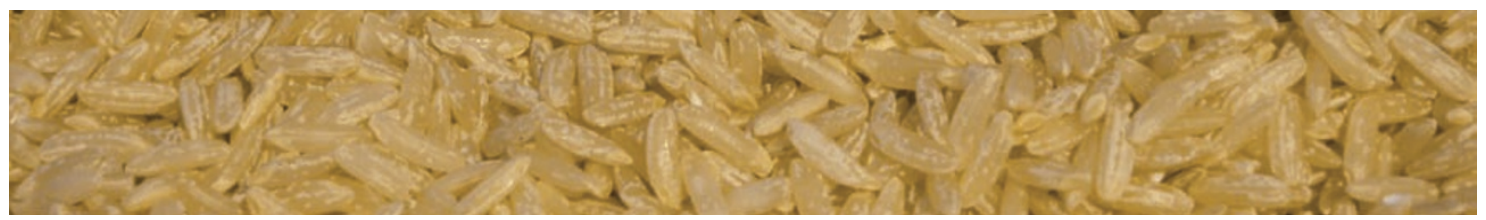

